\title{
Skeletal pigmentation due to tetracycline
}

\author{
H. B. HILTON \\ From the Department of Pathology, Princess Margaret Hospital \\ for Children, Perth, Western Australia
}

SYNOPSIS Two cases of pigmentation of the skeletal system due to tetracycline are reported. It is suggested that tetracycline may be stored in bone due to a tetracycline hydrochloride calciumo phosphate complex after prolonged tetracycline administration, and that tetracycline may be the $\vec{G}$ antibiotic of choice in certain cases of osteomyelitis. Dark pigmentation of the teeth may be due i to prolonged tetracycline therapy and may be the only sign of heavy deposition into the skeletal $\vec{N}$ system.

The side-effects of giving tetracycline include those on the oro-pharyngeal mucous membrane, gastroenteritis, superinfection, anal fissures, vaginitis, drug fever, skin rashes, angio-neurotic oedema, and anaphylaxis. Benign intracranial hypertension has also been reported (Fields, 1961). Dark staining of the teeth has been noted after prolonged administration (Shwachman, Fekete, Kulczycki, and Foley, 1958-59).

The purpose of this paper is to report two cases of skeletal pigmentation due to tetracycline and to discuss its implications.

\section{CASE REPORTS}

CASE 1 P.S., the daughter of English parents, presented at the age of 4 months with symptoms of pulmonary infection. The diagnosis of fibrocystic disease of the pancreas was confirmed by laboratory investigations (sweat sodium $=186 \mathrm{mEq}$./litre; sweat chloride $=223$ $\mathrm{mEq}$./litre). From the age of 4 months to her death at the age of 3 years, she had had repeated attacks of pulmonary infection which necessitated admission to hospital on 15 separate occasions. Antibiotics had been prescribed in large quantities to control pulmonary symptoms and particularly large amounts of tetracycline had been administered during the last year of life (Table I).

There was no evidence of blood dyscrasia. Haemoglobin estimations varied between $11 \mathrm{~g} . / 100 \mathrm{ml}$. and $14 \mathrm{~g}$./ $100 \mathrm{ml}$. and the total leucocyte count varied between $10,000 / \mathrm{c} . \mathrm{ml}$. and $19,600 / \mathrm{c} . \mathrm{ml}$. The lowest neutrophil count recorded was $2,000 / \mathrm{c} . \mathrm{ml}$. Radiographs of the chest, including one taken one week before death, demonstrated no abnormality of vertebrae or ribs. A radiograph of the right arm taken one year before death showed no abnormality of the humerus, radius, ulna or wrist bones.

Received for publication 10 August, 1961.
The main features noted at necropsy were purulent bronchiectasis, bronchopneumonia, and pulmonary $\widehat{\supset}$ emphysema, cardiac enlargement due to right ventricular hypertrophy, and evidence of cor pulmonale.

The liver was pale due to fatty degeneration, and mul- $N$ tiple small, depressed, bile-stained areas were present 0 immediately beneath the liver capsule. The gall bladder was hypoplastic.

The pancreas was firmer and more granular than normal, suggesting fibrocystic disease of the pancreas.

The urogenital system and central nervous system 0 showed no abnormality.

All bones, which included the skull, ribs, vertebrae, sternum, pelvis, femur (Fig. 1), and humerus, examined at necropsy were a striking yellow colour. The yellowcolour was obvious on superficial examination of the flat bones but only seen in the femur and humerus after용 removal of the periosteum. Pigmentation extended: through the whole thickness of the cortex and involved 3 . the bony trabeculae of the medullary cavity. The bone marrow was red. Cartilage was not affected. No organs apart from the skeletal system showed evidence of pigmentation. The teeth were not pigmented.

Histological findings The diagnosis of fibrocystic disease of the pancreas was confirmed by the typical histological findings.

Sections through the subcapsular bile-stained areas of the liver showed proliferation of bile ducts within the $N$

TABLE I

TOTAL QUANTITY OF ANTIBIOTICS GIVEN TO CASE 1

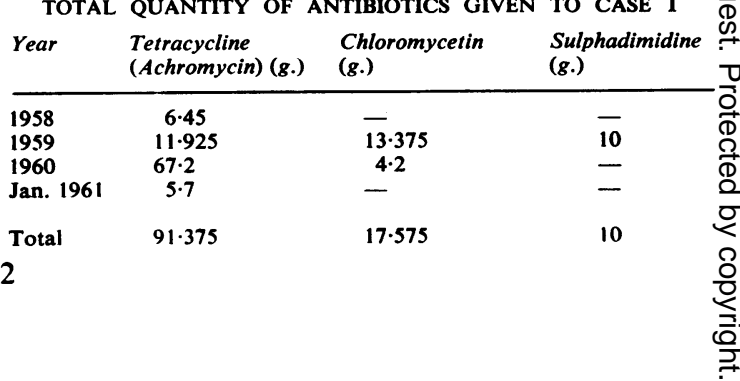




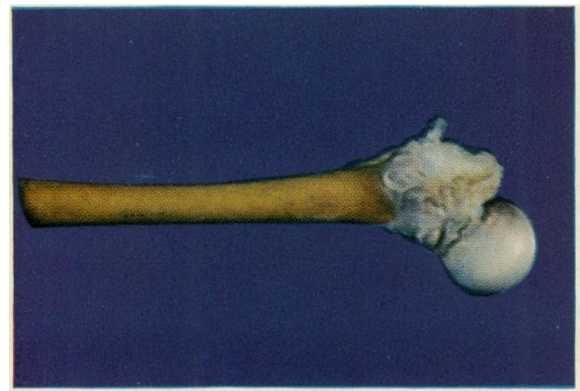

FIG. 1 .

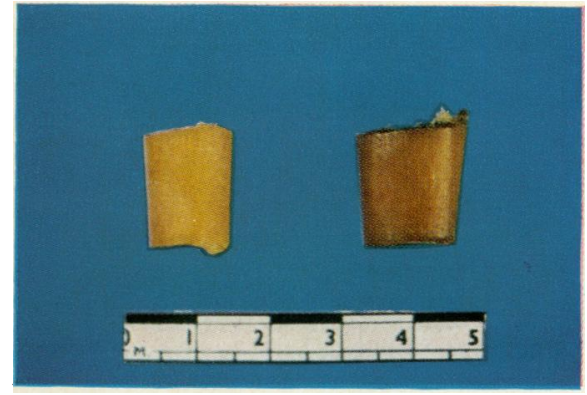

FIG. 1. Yellow pigmentation of the femur. Cartilage is not pigmented.

FIG. 2. Before and after exposure to light. The yellow bones gradually became brown on exposure to light.

FIG. 2 . portal tracts. Many of the bile ducts were filled with eosinophilic material.

Section of the rib revealed no abnormality. The bone marrow was normally cellular.

CASE 2 A girl presented at the age of 28 months with cough, fever, malaise, and pallor. Hepatosplenomegaly was discovered on examination and blood investigations revealed anaemia and neutropenia (haemoglobin 4.5 g./100 ml.; W.B.C. 4,200/c.ml. with $97 \%$ lymphocytes and $3 \%$ neutrophils). A bone marrow examination showed $45 \%$ of the cells to be of reticulo-endothelial type.

Treatment included blood transfusions, cortisone, and antibiotic therapy. During the illness approximately $25 \mathrm{~g}$. of tetracycline hydrochloride was administered. The child's condition gradually deteriorated and death occurred six months after the first appearance of symptoms. Terminally, primitive reticulo-endothelial cells appeared in the peripheral blood and the diagnosis of leukaemic reticulo-endotheliosis was suggested.

The main findings at necropsy included hepatosplenomegaly, enlarged mesenteric lymph nodes, and massive infiltration of the kidneys, pancreas, and lungs.

The inner surface of the skull was a uniform yellow colour. Pigmentation of the outer surface was not marked but yellow coloration along the suture lines was obvious. The ribs were pale yellow and a slight yellowish tinge was seen in the femur, clavicles, and vertebrae. Cartilage was not affected. The teeth were not pigmented.

The cortex of all bones was thinner than normal and pale reddish marrow was present in all bones examined.

Histological findings The bone marrow was replaced by reticulo-endothelial cells and the bony cortex was thinned but otherwise normal.

Monocytic and reticulo-endothelial cells had penetrated the kidneys, spleen, lungs, pancreas, liver, and lymph nodes.

\section{INVESTIGATION OF BONE PIGMENT}

ACTION OF LIGHT It was observed that the yellow bones gradually became brown on exposure to light. That the colour change was caused by exposure to light was suggested by the following simple experiment. Two pieces of yellow femur were taken, one piece was exposed to air and light, the other piece to air but not to light. The piece of bone exposed to light became brown, the bone not exposed to light remained yellow (Fig. 2).

FLUORESCENCE IN ULTRA-VIOLET LIGHT The yellow bones were shown to fluoresce in ultra-violet light. After exposure to light the brown bones failed to fluoresce. Tetracycline hydrochloride (Lederle) fluoresced but processed tetracycline (see below) and extract of normal bone failed to fluoresce.

ULTRA-VIOLET SPECTRA The yellow pigment was extracted from bone by the following method:

The ground bone was dissolved in N. hydrochloric acid, the solution filtered and evaporated, the residue suspended in hot ethyl alcohol, and the undissolved matter filtered off and discarded. The filtrate was evaporated, the residue suspended in hot ethyl alcohol, and the undissolved residue filtered off and discarded. The filtrate was again evaporated to dryness leaving a brown residue.

A sample of tetracycline hydrochloride (Lederle) and a normal bone was treated in a similar manner. The ultra-violet spectra of the extracted pigment, unprocessed tetracycline hydrochloride, processed tetracycline hydrochloride, and extract of normal bone was determined in a Unicam spectrophotometer 500 against $\mathrm{N}$. hydrochloric acid as a blank (Figs. 3 and 4).

In the ultra-violet spectra of processed tetracycline hydrochloride, unprocessed tetracycline hydrochloride, and extracted bone pigment an absorption peak was present at $270 \mathrm{~m} \mu$. The absorption peak at $351 \mathrm{~m} \mu$ present in the unprocessed tetracycline hydrochloride was absent from the processed tetracycline hydrochloride and extract of bone pigment.

An absorption peak was present at $220 \mathrm{~m} \mu$ in the ultra-violet spectra of both the processed and unprocessed tetracycline hydrochloride. The high absorption peak up to $230 \mathrm{~m} \mu$ seen in the ultra-violet spectra of both normal bone and extracted bone pig- 


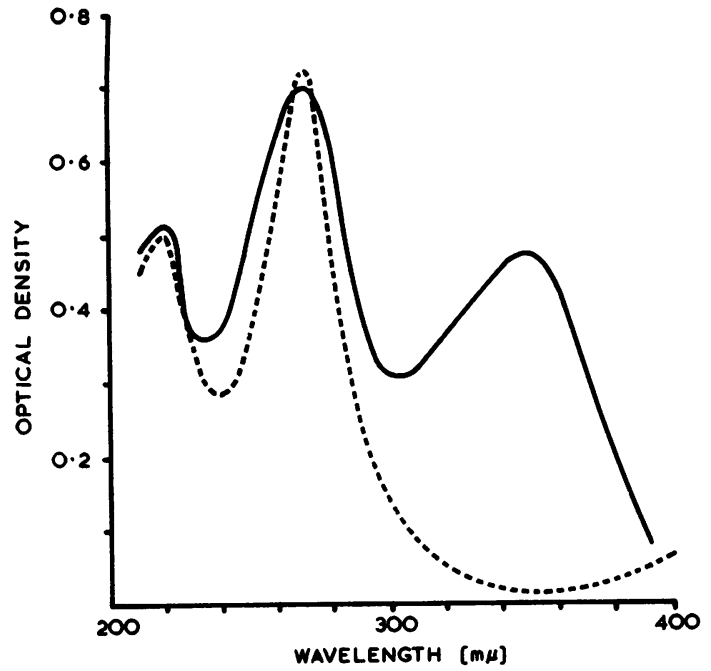

FIG. 3.

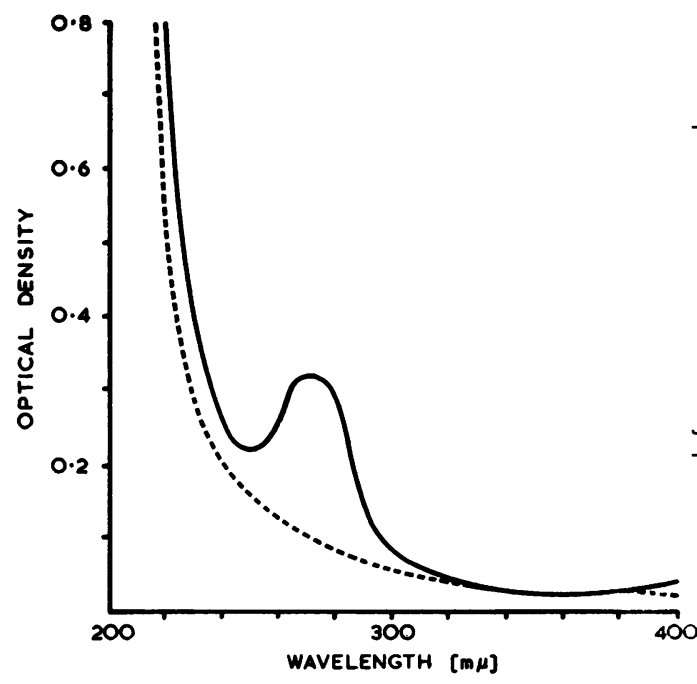

FIG. 4.

FIG. 3. Ultra-violet spectra of tetracycline hydrochloride (continuous line) and processed tetracycline hydrochloride (broken line). The absorption peak at $351 \mathrm{m \mu}$ in the unprocessed tetracycline hydrochloride is absent from the processed tetracycline hydrochloride.

FIG. 4. Ultra-violet spectra of extracted bone pigment (continuous line) and extract of normal bone (broken line) The absorption peak at $270 \mathrm{m \mu}$ in the extracted bone pigment is absent from the extract of normal bone.

ment would obscure any absorption peak between 200 and $230 \mathrm{~m} \mu$ if present.

ANTIBACTERIAL ACTIVITY OF EXTRACTED PIGMENT The antibacterial activity of tetracycline hydrochloride (Lederle), processed tetracycline hydrochloride, and extracted bone pigment was compared, using sensitive and resistant bacteria. The antibacterial activity of both extracted bone pigment and processed tetracycline hydrochloride were practically identical and similar to that of the unprocessed tetracycline hydrochloride though in lesser degree.

AFFINITY OF TETRACYCLINE HYDROCHLORIDE FOR CALCIUM PHOSPHATE It was shown by a simple test tube technique that when calcium orthophosphate was precipitated from an aqueous solution, which also contained tetracycline hydrochloride, the tetracycline hydrochloride was absorbed on to the precipitate. The same phenomenon occurred with magnesium hydroxide and to a slight extent with calcium hydroxide but not with magnesium orthophosphate. It is suggested that a tetracycline hydrochloride-calcium orthophosphate complex may occur.
DISCUSSION

After oral ingestion, tetracycline diffuses rapidly throughout body fluids (Finland, Purcell, Wright Del Love, Mou, and Kass, 1954; English, P'an McBride, Gardocki, Van Halsema, and Wrighto 1953-54; Maynard, Andriola, and Prigot, 1953-54 Wood and Kipnis, 1953-54). Therapeutic serum levelș are rapidly attained, and significantly high serum level§ persist for 48 hours after administration has ceased (Milberg, Kamhi, and Banowitch, 1954). Tetra cycline appears rapidly in the urine but poor renals function is associated with a low urinary excretion (Milberg et al., 1954). A small amount of tetracycline hydrochloride is excreted in the bile.

Studies of the distribution of tetracycline in the tissues of the mouse by means of autofluorescences microscopy have shown the highest concentrationso of these drugs to be in the liver, the kidney, and the reticulo-endothelial system (Helander and Böttiger 1953; Böttiger, 1955). Snell, Garkuscha, and Allere? (1957-58) found the greatest accumulation of tetra-0 cycline in the livers of mice, and Leevy, Zinke, and Chey (1958-59) found tetracyclines to be sequestereक in the liver in man.

The reported urinary recovery rates of tetracycline vary considerably. Finland et al. (1954) recovered 
9 to $21 \%$ of an ingested $1 \mathrm{~g}$. dose of tetracycline in the first 12 hours after administration, and 4 to $7 \%$ in the second 12-hour period. Sweeney, Hardy, Dornbush, and Ruegsegger (1957) recovered 30 to $40 \%$ of orally administered tetracycline in the urine. Kunin, Dornbush, and Finland (1959) found that the average 96-hour urinary recovery of tetracycline was $60 \%$ of the intravenously administered dose of $500 \mathrm{mg}$.

In our first case the urogenital system was normal and lack of urinary excretion was probably not a feature. The liver showed some evidence of bile duct hyperplasia and obstruction as is often found in this disease, and the possibility of decreased biliary excretion cannot be ignored. In Case 2 massive leukaemic infiltration of the liver and kidneys was present.

Yellow pigmentation of bones of dogs and rats following tetracycline and chlortetracycline has been previously noted (Dessau, cited by Buyske, Eisner and Kelly, 1960). Buyske et al. (1960) noted a cumulative deposition of tetracycline and chlortetracycline in bones of rats on long-term drug and diet régimes but no deleterious effects were recorded. A fluorophor localization in bone, particularly in growing bone and malignant bone tumours, has been reported (Rall, Loo, Lane, and Kelly, 1957; Milch, Rall, and Tobie, 1957), and McLeay and Walske (1960) observed brilliant yellow fluorescence under ultra-violet light in malignant bone tumours after intravenous tetracycline.

Shwachmanetal.(1958-59) observed the occurrence of dark staining of teeth in 40 out of 50 children suffering from fibrocystic disease of the pancreas while they were having prolonged therapy with tetracycline. We have observed the darkening of pigmented bones on exposure to light and suggest that the dark staining of the teeth observed by
Shwachman could be attributed to deposition of tetracycline. Necropsy studies on these patients were not reported.

In Case 1 the gross deposition of tetracycline in bone was not associated with any blood dyscrasia. This tends to confirm the lack of toxicity of tetracycline for bone marrow. Storage in bone in high concentration suggests that tetracycline may be the drug of choice in certain cases of osteomyelitis, providing the infecting organism is sensitive to it.

I wish to thank Mr. E. Edkins for technical help, Dr. N. Barsden for the notes of Case I, and Professor W. B. Macdonald for the notes of Case 2.

\section{REFERENCES}

Böttiger, I . E. (1955). Acta med. scand., 151, 343.

Buyske, D. A., Eisner, H. J., and Kelley, R. G. (1960). J. Pharmacol. exp. Ther., 130, 150.

English, A. R., P'an, S. Y., McBride, T. J., Gardocki, J. F., Van Halsema, G., and Wright, W. A. (1953-54). Antibiot. Ann., p. 70.

Fields, J. P. (1961). J. Pediat., 58, 74.

Finland, M., Purcell, E. M., Wright, S. S., Del Love, B., Mou, T. W., and Kass, E. H. (1954). J. Amer. med. Ass., 154, 561.

Helander, S., and Böttiger, L. E. (1953). Acta med. scand., 147, 71.

Kunin, C. M., Dornbush, A. C., and Finland, M. (1959). J. clin. Invest., 38, 1950.

Leevy, C. M., Zinke, M. R., and Chey, W. Y. (1958-59). Antibiot. Ann., p. 258.

Maynard, A. de., Andriola, J. C., and Prigot, A. (1953-54). Ibid., p. 102.

McLeay, J. F., and Walske, B. R. (1960). J. Bone Jt Surg., 42-A, 940.

Milberg, M. B., Kamhi, Bernard, and Banowitch, M. M. (1954). Antibiot. and Chemother., 4, 1086.

Milch, R. A., Rall, D. P., and Tobie, J. E. (1957). J. nat. Cancer Inst., $19,87$.

Rall, D. P., Loo, Ti Li, Lane, M., and Kelly, M. G. (1957). J. nat. Cancer Inst., 19, 79.

Snell, J. F., Garkuscha, R., and Allen, E. L. (1957-58). Antibiot. Ann., p. 502.

Shwachman, H., Fekete, E., Kulczycki, L. L., and Foley, G. E. (1958-59). Ibid., p. 692.

Sweeney, W. M., Hardy, S. M., Dornbush, A. C., and Ruegsegger, J. M. (1957). Antibiot. Med., 4, 642.

Wood, W. S., and Kipnis, G. P. (1953-54). Antibiot. Ann., p. 98. 